\title{
Artisanal Cannabis in Young and Adults Patients with Treatment Resistant Epilepsy: Prospective, Observational, Open-Label Study in Argentina
}

\author{
Kochen $\mathrm{S}^{* 1,2}$, Manin $\mathrm{A}^{1}$, and Montiel $\mathrm{R}^{2}$ \\ ${ }^{1}$ Epilepsy Center, Department Neurology, Hospital “R. Mejía”, University Buenos Aires, Argentina \\ ${ }^{2}$ Neurosciences and Complex Systems Unit (EnyS), CONICET-Hospital El Cruce Néstor Kirchner-National Univer- \\ sity, A. Jauretche (UNAJ), Argentina
}

*Corresponding author: Kochen S, Epilepsy Center, Department Neurology, Hospital “R.Mejía", University Buenos Aires, Argentina, Neurosciences and Complex Systems Unit (EnyS), CONICET-Hospital El Cruce Néstor Kirchner-National University, A. Jauretche (UNAJ), Argentina, Tel: +54 11 42109000, E-mail: skochen@gmail.com

Citation: Kochen S, Manin A, Montiel R (2018) Artisanal Cannabis In Young And Adults Patients With Treatment Resistant Epilepsy: Prospective, Observational, Open-Label Study In Argentina. J Neurol Neurol Disord 4(2): 202. doi: 10.15744/2454-4981.4.202

Received Date: June 18, 2018 Accepted Date: July 13, 2018 Published Date: July 16, 2018

\begin{abstract}
Background: A third of patients with epilepsy have a drug-resistant form. We aimed to observed whether addition of artisanal cannabis to existing anti-epileptic treatment would be safe, and efficacious in young and adults with drug-resistant epilepsy.

Methods: Patients (aged 12-51 years) with severe, intractable epilepsy, who were receiving stable doses of antiepileptic drugs before study entry, were enrolled in an expanded-access programme at our epilepsy centre. Patients took whole plant hemp extract containing cannabinoids, and THC levels of $0.3 \%$ or less. The primary efficacy endpoint was median percentage change in the mean monthly frequency of seizures at 24 weeks.

Results: 92 patients were enrolled, 52 patients who had at least 24 weeks of follow-up were included in the analysis. The most common epilepsy treated was Lennox-Gastaut syndrome. Adverse events were reported in 14 (26.9\%), no serious adverse events. Baseline median monthly frequency of seizures was 24 /week and decreased to 19,05 /week. Seven (13.46\%) patients were free of all seizures. Median change in total seizures was $-66.6 \%$ (IQR-100 to $-48,3$ ).

Conclusion: Our findings suggest in an older group that previously published, that cannabidiol might reduce seizure frequency and might have an adequate safety profile. Randomised controlled trials are necessary for to establish safety profile and efficacy of cannabis.

Keywords: Epilepsy; Cannabis; Open-label; Drug resistant Epilepsy; Efficacy
\end{abstract}

\section{Introduction}

Despite the availability of more than 20 different drugs for epilepsy (AEDs) and the provision of appropriate medical therapy, 30\% of patients with epilepsy continue to have seizures [1,2]. Including the new drugs during the past two decades has not reduced the proportion of patients with drug resistant epilepsy [1]. The safety and side-effect profile of AEDs has improved, but side effects related to the central nervous system are common and affect quality of life [3]. Patients need new treatments that control seizures and have fewer side effects. This limitation of treatment has led patients and families to seek alternative treatments. Cannabisbased treatment for epilepsy has recently received prominent attention in the lay press and in social media, in the world as in our country, with reports of dramatic improvements in seizure control in patients with severe epilepsy [4].

In response, last March in Argentina the Parliament approved a law supported by all their members that have legalized cannabis medicinal (https://www.boletinoficial.gob.ar/\#!DetalleNorma/162454/20170419. Ley 27350. Uso Medicinal de la Planta de Cannabis y sus derivados). Cannabis has been used medicinally for millennia and was used in the treatment of epilepsy as early as 1800 b.c.e. in Sumeria [5]. The use of cannabis therapy for the treatment of epilepsy diminished with the introduction of phenobarbital (1912) and phenytoin (1937) and the passage of the Marijuana Tax Act (1937). The discovery of an endogenous cannabinoid-signaling system in the 90's renewed interest in therapies derived from constituents of cannabis for nervous system disorders such as epilepsy [6]. 
The current preclinical and clinical data that suggest that compounds found in cannabis have efficacy against seizures. More than 545 distinct compounds have been isolated from cannabis species; the most abundant are the cannabinoids, a family of molecules that have a 21-carbon terpenophenolic skeleton and includes numerous metabolites [7]. Cannabis contains more than 80 phytocannabinoids, termed "phytocannabinoids" if derived from the plant, the best studied of these cannabinoids, are two compounds are $\Delta 9$-tetrahydrocannabinol $(\Delta 9$-THC) and cannabidiol and their metabolites. Most of the psychoactive effects of cannabis are mediated by $\triangle 9$-THC, has been shown to have both anticonvulsant and proconvulsant effects in animal studies of seizures [8].

Preclinical evidence showing the anticonvulsant properties of cannabidiol in animals supports this focus on preparations with high cannabidiol content [8,9]. Furthermore, recently trials in humans, an open-label trial and other double-blind, placebocontrolled trial, suggested benefits in seizure control and good tolerability, with patients experiencing mild or no side-effects $[10,11]$. Also in recent times Epilepsy \& Behavior produced a special issue that presents an in-depth assessment of the potential of cannabinoids for the effective treatment of epilepsy [12].

Given the the intense interest from our patients and their families, most young adults with severe epilepsy in obtaining cannabis, we did this study to test whether cannabidiol as an add-on treatment to conventional anti-epileptic drugs would be safe, tolerated, and efficacious in young adults and adults with highly drug -resistant epilepsy.

\section{Methods}

\section{Study Design and Patients}

We did this prospective, observational, open-label, expanded-access study at our Epilepsy Centre, Div. Neurology, Hosp. "R. Mejía” in Buenos Aires City. Inclusion criteria for entry into some of two expanded-access programme, patients were aged for patients 12 years and older, had intractable epilepsy, had five or more countable seizures per 4 week period, and were receiving stable doses of antiepileptic drugs for at least 4 weeks before enrolment. All the patients included in this study had a minimum follow-up of 6 months. All patients were taking more than two antiepileptic drugs, some up six drugs (Table 1). Exclusion criteria included previous or current treatment with cannabis-based therapy. Also excluded patients with baseline liver, renal, or haematological laboratory abnormalities.

\begin{tabular}{|c|c|}
\hline Clinical features of patients & Efficacy analysis group (n:52) \\
\hline \multicolumn{2}{|c|}{ Sex } \\
\hline Female & $22(42,30 \%)$ \\
\hline Male & $30(57,69 \%)$ \\
\hline Age (Years) & $11-51($ mean 28$)$ \\
\hline Background antiepileptic drugs & $3(0-7)$ \\
\hline Total daily treatments & $3(1-7)$ \\
\hline $\begin{array}{c}\text { Receiving vagal nerve stimula- } \\
\text { tion }\end{array}$ & $4(8 \%)$ \\
\hline On a ketogenic diet (\%) & $3(6 \%)$ \\
\hline Receiving clobazam (\%) & $10(20 \%)$ \\
\hline Receiving valproate (\%) & $15(29 \%)$ \\
\hline \multicolumn{2}{|c|}{ TOTAL SEIZURES } \\
\hline Weekly frequency & Mean $45(0,5-420)$ \\
\hline
\end{tabular}

Parents or patients provided written informed consent, and assent according to patients' physical and mental capability.

\section{Procedures}

After enrolment patients had a 4 week pre-cannabidiol baseline period during which parents or caregivers kept prospective seizure diaries. They were asked to report countable seizures, easier to counting as tonic-clonic, tonic, clonic, atonic, and focal seizures. Patients chose depending on the availability if they used one or another formulation. After the observation period, patients taking a non-purified cannabis preparations from Charlotte's Web ${ }^{\text {tw }}(\mathrm{CW})$ (https://www.cwhemp.com), or taking a non-purified cannabis preparations from cannabicultors local of CAMEDA (http://www.cannabismedicinal.com.ar).

Both prepared, according to report of cannabicultors, are a whole plant hemp extract containing cannabinoids, and THC levels of $0.3 \%$ or less, in a $50 \mathrm{mg}$ per $\mathrm{mL}$ sesame oil-based solution cannabidiol. For patients who received CW, content of cannabinoids in the extract tested and validated by a laboratory approved in local producers. For patients who received from CAMEDA content of cannabinoids in the extract tested and validated by a laboratory approved by University in Argentina. The government of 
Argentine through of ANMAT (National Administration of Drugs, Foods and Medical Devices) authorized the importation of CW as dietary supplement. Administered orally was used with both preparation. Patients took what would be the equivalent of a dose of $2,5 \mathrm{mg} /$ per day to $50 \mathrm{mg} /$ per day divided in twice- or three daily dosing was added to the baseline antiepileptic drug regimen, then up-titrated by $2-50 \mathrm{mg} /$ day once a week until intolerance or a maximum dose of $22,5 \mathrm{mg} / \mathrm{per}$ day to $650 \mathrm{mg} /$ per day was reached. This expanded difference of dose is due to the fact that patients followed recommendations of the $\mathrm{CW}$ or cannabicultors local.

All seizures were recorded by parents or caregivers on paper diaries and reviewed by the study team at each clinic visit. Tolerability and adverse effects were assessed every 2 weeks, we use as guide the Liverpool Adverse Events Profile [13].

Additional variables measured prospectively in patient diaries were use of rescue medications, episodes of status epilepticus, and emergency room visits or hospital admissions. Laboratory studies for haematology, electrolytes, liver and kidney function, and some patients concentrations of clasic antiepileptic drugs (PHB, PHT, CBZ and Valproic Acid).

\section{Outcomes}

The primary endpoint was to establish the safety and the primary efficacy of cannabidiol, outcome was median percentage change in the mean monthly frequency of seizures at 24 weeks. Mean monthly seizure frequency was calculated at each 4 week visit as (number of seizures since last visit)/(days since last visit) $\times 28$. Median percentage change was calculated because patients had a wide range of seizure frequencies. Percentage change in seizure frequency for each patient was calculated based on seizures can be more reliably counted by observers than subjective or transient events, most of whom a degree of intellectual disability or epileptic encephalopathy had. Thus, this endpoint was chosen in view of concern about potential parental bias due to media attention and desire for the patient to receive cannabidiol. We also examined the response rate for all seizure types, defined as patients whose reduction in mean monthly seizure frequency was free of seizures, greater than $50 \%$, lesser than $50 \%$, equal or worst over the study observation period. Response rate was calculated from individual patient percentage reductions. Because of the long titration phase for cannabidiol, we examined the median percentage change in seizure frequency during the final 4 weeks of the observation period.

\section{Statistical Analysis}

The study sample size was not predetermined, but based on patient enrolment at the study. Comparisons of the percentage change in frequency of seizures were done with a Mann-Whitney U test. We did analyses with SPSS (version 20).

\section{Results}

Between March 1, 2016, and June 1, 2017, 92 patients were enrolled in expanded-access programmes. Only 52 patients were included in the analysis, met our inclusion and exclusion criteria for safety and efficacy analysis. In the last time we enrolled 27 patients, but not had six months of follow up, and 13 subjects were excluded because, 12 had aged $<12$ year, and one patient had no treatment-resistant epilepsy. There were no differences between CW or cannabicultors local groups, (27 and 25 patients respectively) neither demographic nor in the clinical characteristics. We found no differences in efficacy or in the presence of adverse effects, we present the results of both groups together.

Only one patient stopped treatment before 24 weeks for adverse effect, hypotension. Table 1 shows the baseline clinical and demographic characteristics of patients. The most common epilepsy treated were Lennox-Gastaut syndrome (Table 2). Adverse events were reported in 14 (26.9\%) of the 52 patients. Events reported in more than $5 \%$ of patients were somnolence, fatigue, and irritability. Most adverse events were mild or moderate and transient (Table 3). Additionally, the relatives or patients reported somnolence and fatigue at start a new increased of dose, but or they reduced the dose or divised the entire dose, but it was not possible for us to establish a correlation with cannabidiol dose. Also probably somnolence it was in relation with THC of the preparation.

\begin{tabular}{|c|c|}
\hline & Safety analysis group (N:52) \\
\hline Lennox-Gastaut syndrome & $9(17,3 \%)$ \\
\hline Other & $17(32 \%)$ \\
\hline Unknown & $8(15,3 \%)$ \\
\hline Epileptic encephalopathy & $6(11,5 \%)$ \\
\hline West syndrome & $3(5,7 \%)$ \\
\hline Febrile infection-related epilepsy syndrome & $3(5,7 \%)$ \\
\hline Tuberous sclerosis complex & $2(3,84 \%)$ \\
\hline
\end{tabular}

Table 2: Epilepsy syndromes and underlying causes 


\begin{tabular}{|c|c|}
\hline Adverse events & Safety analysis group (N:52) \\
\hline Somnolence & $10(19,2 \%)$ \\
\hline Fatigue & $5(9,61 \%)$ \\
\hline Irritability & $3(5,76 \%)$ \\
\hline Headache & $2(3,84 \%)$ \\
\hline Convulsion & $2(3,84 \%)$ \\
\hline Arterial hypotension & $1(1,92 \%)$ \\
\hline \multicolumn{2}{|c|}{ Table 3: Adverse events }
\end{tabular}

Any serious adverse events were reported. There were no clinically significant changes in white or red blood cell counts or renal function.

Baseline median monthly frequency of seizures was 24 and decreased to 19,05 over the 24 week treatment period (Figure 1). The titration of cannabidiol dose was slow and there was variability between patients, which meant many patients had not stabilised their dose until half way through the 24 week observation period. Thus, we analysed changes in seizure frequency during the final 4 weeks of observation only. During this period 7 (13.46\%) patients were free of all seizures. Post-hoc analysis showed median change in total seizures was $-66.6 \%$ (IQR -100 to -48.3 ) (Figure 2 ).

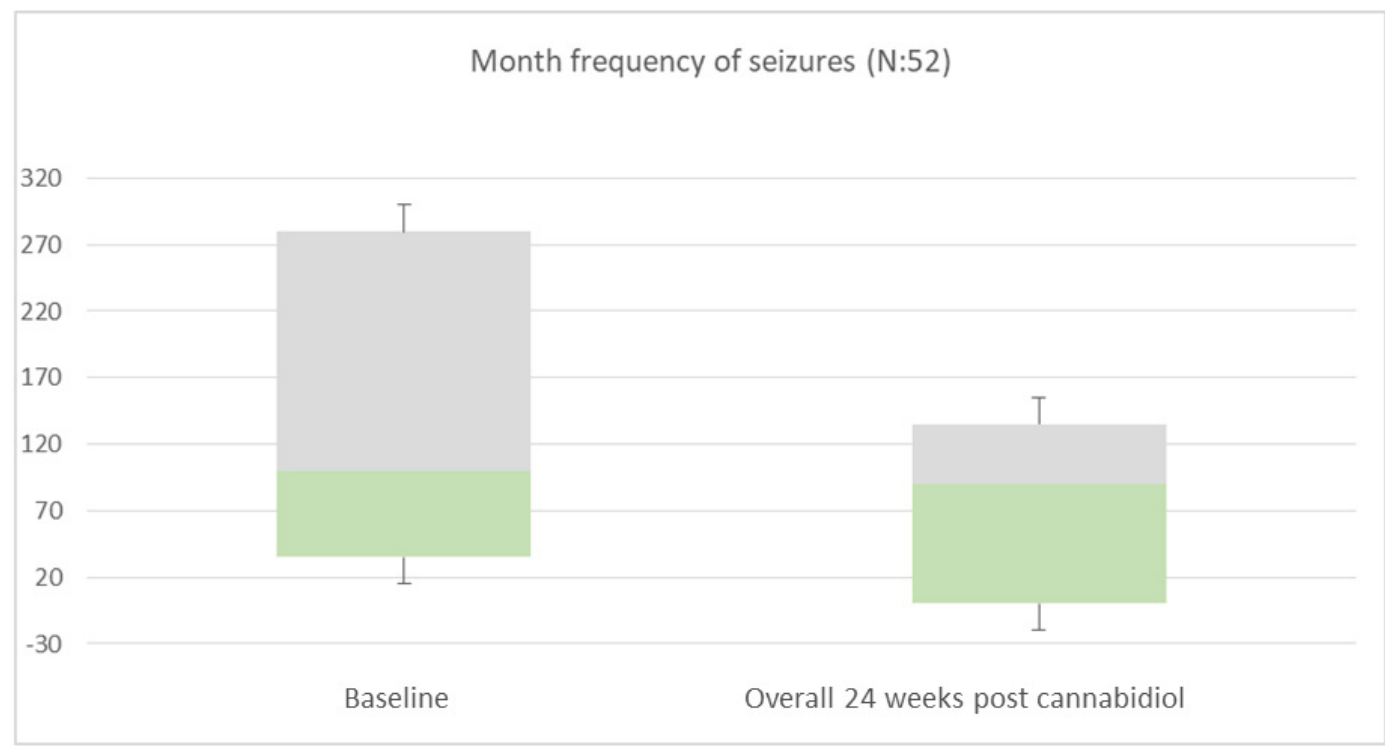

Figure1: Month frequency of seizures (N:52)

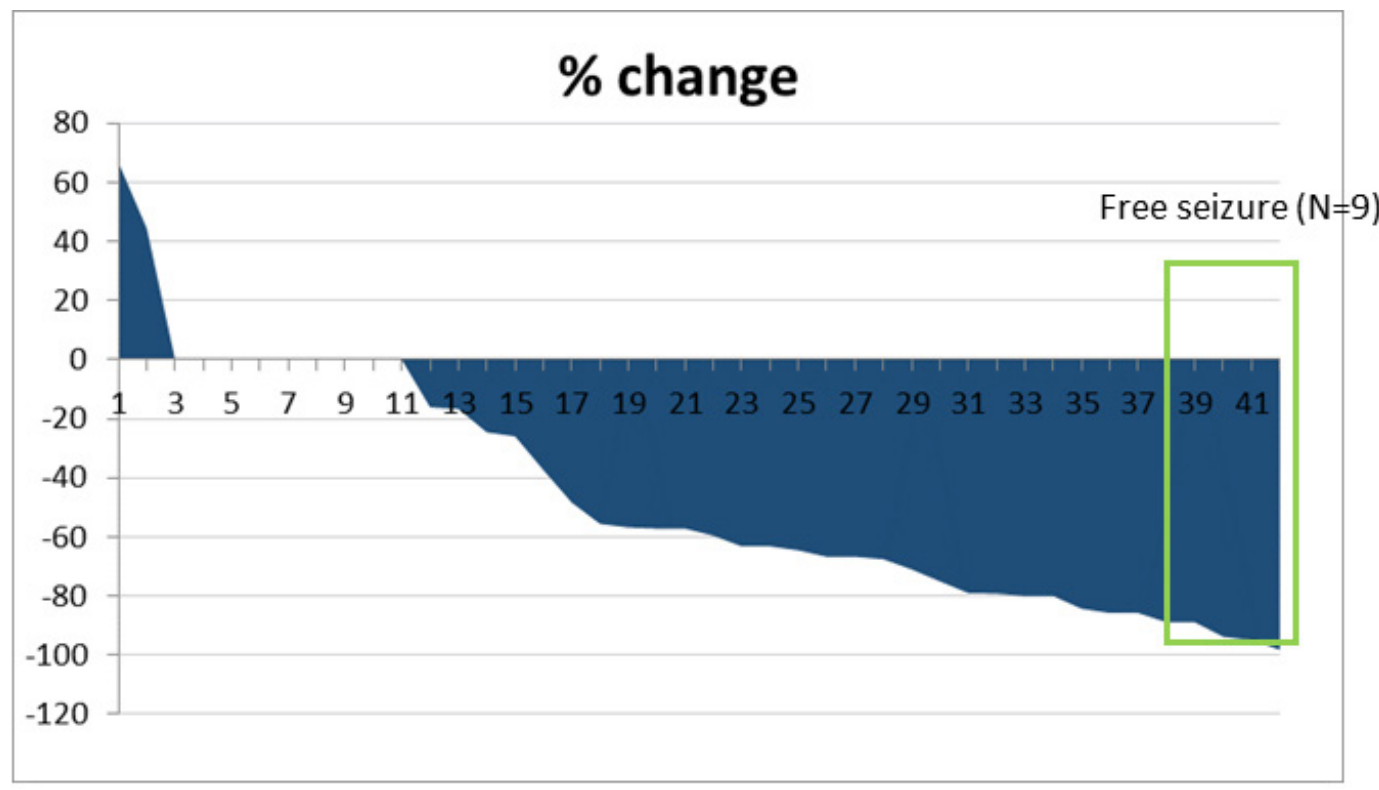

Figure 2: Percentage change in frequency of seizures. Percentage changes for each patient are ordered from greatest increase to greatest decrease 
In relation to dose, we observed the mean cannabidiol dose at 6 months was $51.5 \mathrm{mg}$ per day, maximum dose at the 6 months was $750 \mathrm{mg}$ per day and minimum dose was $5 \mathrm{mg} /$ per day. Additional post-hoc analysis showed that cannabidiol dose at week 24 was not correlated with the number of reported adverse events overall. Additionally, there was no correlation with cannabidiol dose and reported frequency of seizures.

We observed no differences in efficacy outcomes or adverse effects reported in the group of patients taking the preparation of $\mathrm{CW}$ or cannabicultors local. Almost $70 \%$ of patients or their relatives refered us an improvemet in relation to sleep and more connected with the social environment.

\section{Discussion}

In our open-label study, add-on treatment with cannabidiol led to a clinically meaningful reduction in seizure frequency in many patients, and had an adequate safety profile in this patient population with highly treatment-resistant epilepsies. Only one patient of 52 patients stopping treatment because of an adverse event. Common adverse events included somnolence, fatigue, and irritability. Somnolence and fatigue could be partly due to presence of THC. The efficacy of cannabidiol seems promising, with median change in total seizures was $-66.6 \%$ (IQR -100 to -48.3 ) overall seizures, and 7 (13.46\%) patients were free of all seizures. However, randomised controlled trials are needed to characterise the safety profile and true efficacy.

Patients included in this study had syndromes characterised by poor prognosis. Many of these patients had never achieved complete seizure control despite treatment with antiepileptic drugs, vagus nerve stimulation. The median number of concomitant antiepileptic drugs as three. We observed high rates of seizure reduction, and even seizure freedom for a few patients, suggest clinically meaningful efficacy. However, without a control group, the results regarding safety and efficacy must be interpreted cautiously.

While open label studies have been published a number of times for CBD enriched preparations, it is a first study from a non US or Isreali group would be useful addition to the literature. In addition, this study includes in an older group that previously published.

Cannabis has been used as a therapeutic agent since ancient times. Its use slowly declined, may be due to the constituents of Cannabis had not been isolated in a pure form. And for legally, in most countries, the use of these drugs was officially controlled and frequently made difficult. Since ' 70 the detailed investigations on THC and other cannabinoids have to some extent clarified the problem of their possible therapeutic potential. From ancient Chinese pharmacopeias to the current Phase III trials of pharmaceutical grade cannabidiol, there has been a dramatic surge in the interest of utilizing cannabis for epilepsy treatment. Yet, access to cannabis for research and therapy is mired in conflicting regulatory policies and shifting public opinion. In next years probably cannabis will be part of treatment for epilepsy.

The major limitations of this study were that open label and uncontrolled. The issue of the placebo response is relevant in trials of cannabis-derived treatments, especially due to media attention and desire for the patient to receive cannabidiol. We expect to make in the next time with the reglamentation of the law, any future research into use of cannabinoids in patients with epilepsy with all best condition since control of cannabidiol, double-blind, placebo-controlled trial, randomised.

\section{Conclusion}

Our findings suggest in an older group that previously published, that cannabidiol might reduce seizure frequency and might have an adequate safety profile. Randomised controlled trials are necessary for to establish safety profile and efficacy of cannabis.

\section{Conflict of Interest}

We have not Conflict of Interest, any Pharmaceuticals Company or ONG's had no role in study design, data analysis, data interpretation, or writing of the report, or in the decision to submit the paper for publication. The corresponding author had full access to all the data in the study and had final responsibility for the decision to submit for publication.

\section{References}

1. Brodie MJ, Barry SJ, Bamagous GA, Norrie JD, Kwan P (2012) Patterns of treatment response in newly diagnosed epilepsy. Neurology 78: 1548-54.

2. Kwan P, Brodie MJ (2000) Early identification of refractory epilepsy. N Engl J Med 342: 314-9.

3. Perucca E, Meador KJ (2005) Adverse effects of antiepileptic drugs. Acta Neurol Scand Suppl 181: 30-5.

4. REEDS (2017) Las reacciones en las redes sobre la sancion de la ley de uso medicinal del cannabis, Argentina.

5. Schultes RE (1973) Man and marihuana. Nat Hist 82: 59.

6. Devane WA, Hanus L, Breuer A, Pertwee RG, Stevenson LA, et al. (1992) Isolation and structure of a brain constituent that binds to the cannabinoid receptor. Science 258: 1946-9.

7. ElSohly M, Gul W (2014) Constituents of cannabis sativa. In: Handbook of cannabis. Oxford University Press, UK.

8. Hill A, Hill T, Whalley B (2013) The development of cannabinoid based therapies for epilepsy. In: Endocannabinoids: molecular, pharmacological, behavioral and clinical features. Bentham Science, Oak Park, Chicago. 
9. Devinsky O, Cilio MR, Cross H, French J, Hill C, et al. (2014) Cannabidiol: pharmacology and potential therapeutic role in epilepsy and other neuropsychiatric disorders. Epilepsia 55: 791-802.

10. Devinsky O, Marsh E, Friedman D, Thiele E, Laux L, et al. (2016) Cannabidiol in patients with treatment-resistant epilepsy: an open-label interventional trial. Lancet Neurol 15: 270-8.

11. Devinsky O, Cross JH, Wright S (2017) Trial of Cannabidiol for Drug-Resistant Seizures in the Dravet Syndrome. N Engl J Med 377: 699-700.

12. Szaflarski JP (2017) Cannabinoids and epilepsy. Epile Behav 70: 277.

13. Panelli RJ, Kilpatrick C, Moore SM, Matkovic Z, D’Souza WJ, et al. (2007) The Liverpool Adverse Events Profi le: relation to AED use and mood. Epilepsia 48: 456-63. 